\title{
Wechsel der Redaktion und Dank an Axel Groenemeyer
}

\author{
Mechthild Bereswill • Anke Neuber
}

Online publiziert: 21. Juni 2017

(C) Springer Fachmedien Wiesbaden GmbH 2017

\section{Liebe Leser*innen,}

das Heft 1/2017 der Zeitschrift Soziale Probleme ist die erste Ausgabe, an der Axel Groenemeyer nicht mehr als Redakteur mitgewirkt hat. Anke Neuber (Universität Kassel) hat ihn im Oktober 2016 abgelöst, so dass die Redaktion nun in ihren und in den Händen von Mechthild Bereswill (Universität Kassel) liegt.

Im Namen der Redaktion, des Herausgeber*innenkreises und des alten und neu gewählten Vorstandes der Sektion Soziale Probleme in der Deutschen Gesellschaft für Soziologie (DGS) danken wir Axel Groenemeyer ganz herzlich für sein langjähriges Engagement! Unsere Zeitschrift wird weithin wahrgenommen und geschätzt, das zeigen nicht zuletzt die zahlreichen Beiträge, die bei der Redaktion eingereicht werden und fortlaufende Initiativen für Schwerpunkthefte. Dass die Zeitschrift Soziale Probleme eine attraktive Adresse für Autor*innen ist und Leser*innen eine anregende wissenschaftliche Lektüre bietet, ist maßgeblich dem Einsatz von Axel Groenemeyer zu verdanken. Wir freuen uns deshalb sehr, dass er weiterhin im Herausgeber*innenkreis der Zeitschrift mitwirkt und seine Erfahrungen und Perspektiven auf diesem Weg in die weitere Entwicklung der Zeitschrift einfließen.

M. Bereswill $(\bowtie) \cdot$ A. Neuber

Universität Kassel, Kassel, Deutschland

E-Mail: bereswill@uni-kassel.de

A. Neuber

E-Mail: neuber@uni-kassel.de 
Das vorliegende Heft 1/2017 der Zeitschrift Soziale Probleme umfasst sechs Beiträge in einem breiten Themenspektrum von gesellschaftstheoretischen Debatten bis hin zu klassischen empirischen Untersuchungsfeldern der Soziologie sozialer Probleme und der Kriminologie. Wir wünschen viel Vergnügen bei der Lektüre und freuen uns über Rückmeldungen.

Mechthild Bereswill

Anke Neuber 